\title{
Patrón de fracturas óseas en accidentes de motocicleta en Hospital de Alta Especialidad
}

\author{
Pattern of bone fractures in motorcycle accidents in a High Specialty Hospital \\ Ramos-Villalón SA,* Vázquez-López E, ‡ Damián-Pérez R,§ López-Estrada D, " Díaz-Zagoya JC" \\ Hospital Regional de Alta Especialidad «Dr. Gustavo A Rovirosa Pérez», Villahermosa, Tabasco.
}

RESUMEN. Introducción: Los accidentes en motocicleta son una de las causas principales de muerte e incapacidad en la población joven de México. No hay estudios locales que describan el patrón de lesiones con base en la clasificación AO. Objetivo: Definir el patrón y tipo de fracturas óseas más frecuentes atendidas en un Hospital Regional de Alta Especialidad, de Enero a Agosto de 2017. Material y métodos: Estudio descriptivo, transversal, observacional, cuantitativo de 98 pacientes que sufrieron accidente en motocicleta y que ameritaron tratamiento ortopédico quirúrgico ( $94 \%$ hombres, 6\% mujeres, media de edad 29.7 años). Resultados: Los accidentes más frecuentes incluyeron las siguientes características: conductores hombres, motocicleta $<150 \mathrm{~cm}^{3}$, área rural, sin uso de casco (75.5\%) y colisión contra un objeto en movimiento. Las fracturas más frecuentes correspondieron a: tibia y peroné (28.9\%), fracturas expuestas (52.3\%), de fémur (25\%) y antebrazo (10.5\%). Considerando la clasificación AO las fracturas más frecuentes fueron AO 42 B 3.3, AO 32A3.2 y AO 23B1.2. Conclusión: Los motociclistas utilizan escasas medidas de protección. El patrón de lesiones evidenció diferencias con lo reportado a nivel nacional. De acuerdo con la clasificación AO, buena parte de los casos corresponden a los de peor pronóstico.

Palabras clave: Accidentes de tránsito en motocicleta, fracturas, luxaciones.
ABSTRACT. Introduction:Motorcycle accidents are one of the leading causes of death and disability in Mexico's young population. There are no local studies describing the pattern of AO-based injuries. Objective: Define the pattern and type of most common bone fractures in a Regional Hospital of High Specialty, from January to August 2017. Material and methods: Descriptive, cross-sectional, observational, quantitative study of 98 patients who suffered motorcycle accidents and who merited surgical orthopedic treatment (94\% men, 6\% women, average age 29.7 years). Results: The most frequent accidents included the following features: male drivers, motorcycle $<150 \mathrm{~cm}^{3}$, rural area, without helmet use $(75.5 \%)$ collision against a moving object. The most common fractures corresponded to: tibia and fibula $(28.9 \%)$, open fractures $(52.3 \%)$, femur $(25 \%)$ forearm (10.5\%). Considering the AO classification the most common fractures were AO 42 B 3.3, AO 32A3.2 and AO 23B1.2. Conclusion: Motorcyclists use few protective measures. The injury pattern showed differences with what was reported nationally. According to the AO rating, much of the cases correspond to those of worst prognosis.

Keywords: Motorcycle traffic accidents, fractures, dislocations.

\section{Nivel de evidencia: IV}

* Especialista en Ortopedia y Traumatología, Hospital Regional de Alta Especialidad «Dr. Gustavo A Rovirosa Pérez», Villahermosa, Tabasco.

‡ Maestro en educación, Tec Milenio, Tecnológico de Monterrey. Ex jefe de Enseñanza del Hospital de Alta Especialidad «Dr. Gustavo A Rovirosa Pérez». Jefe de enseñanza del Hospital Regional Pemex, Villahermosa, Tabasco.

${ }^{\S}$ Médico Especialista, Hospital Regional de Alta Especialidad «Dr. Gustavo A Rovirosa», Villahermosa, Tabasco.

" Profesor del curso de Especialidad en Traumatología y Ortopedia UJAT, Hospital «Dr. Gustavo A Rovirosa Pérez». Ortopedista UNAM. Maestro en Ciencias Médicas UNAM. Cirugía de columna.

" Médico y Profesor, División de Investigación, Facultad de Medicina UNAM, Ciudad de México. Investigador Nacional SNI.

Correspondencia:

Juan C Díaz-Zagoya

División de Investigación, Facultad de Medicina UNAM, Ciudad de México.

E-mail: zagoya@unam.mx

Citar como: Ramos-Villalón SA, Vázquez-López E, Damián-Pérez R, López-Estrada D, Díaz-Zagoya JC. Patrón de fracturas óseas en accidentes de motocicleta en Hospital de Alta Especialidad. Acta Ortop Mex. 2020; 34(6): 376-381. https://dx.doi.org/10.35366/99135

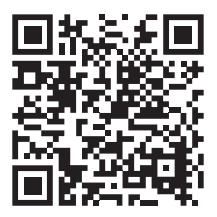




\section{Introducción}

Los accidentes de tránsito constituyen en el mundo la principal causa de muerte no intencionada en jóvenes de 15 y 29 años. ${ }^{1,2}$ Aunque han disminuido (OMS, 2010) siguen siendo un problema que conlleva elementos culturales y generacionales importantes que deben ser esclarecidos.

Con el incremento de la población mundial, ha aumentado la matrícula de motocicletas de diferentes modelos, trayendo consigo la problemática de un sistema de transporte que, gracias a las ofertas en el mercado, puede ser conseguido por los diferentes estratos socioeconómicos, pero que dadas sus características estructurales ha ocasionado que se incrementen los accidentes y muertes en diferentes grupos etarios. ${ }^{3,4}$

En materia de salud, la medicina preventiva debe ser el objetivo principal de un buen médico y no sólo la resolución de una patología. Los accidentes de tránsito son una de las causas del paciente traumatizado, con múltiples fracturas, ${ }^{5}$ que ingresa al Servicio de Urgencias por patología musculoesquelética, por lo que diferentes aristas sobre su caso deben ser entendidas, desde el momento en que sucede el accidente y durante su atención prehospitalaria y hospitalaria.

En el presente estudio, realizado en el Servicio de Urgencias Traumatológicas, se hizo el diagnóstico y la estadística de los individuos lesionados en accidentes de tránsito en motocicleta que fueron recibidos en dicho hospital. Las estadísticas de los accidentes de tránsito en el mundo muestran que de 1990 al 2010 las lesiones por accidentes de tránsito se incrementaron en $46.3 \%$, habiendo 1.3 millones de muertes por año. La mortalidad fue mayor en peatones, seguido de los motociclistas. En este último grupo, en 2010 fue la octava causa de muerte. ${ }^{6}$ En 2015 la Asamblea General de la ONU fijó como una de sus metas para el año 2020 reducir en $50 \%$ el número mundial de muertes y traumatismos por accidente de tránsito, llamando al periodo comprendido del 2010 al 2020 decenio de acción para la seguridad vial. ${ }^{3}$

En la zona geográfica comprendida en este estudio, el parque vehicular en el 2014 fue de 513,433, de los cuales 13\% eran motocicletas; en el año 2016 el porcentaje de motocicletas subió a $14.7 \%$ y la tasa de accidentes por cada 1,000 motocicletas fue de 40.18, según cifras del Centro Nacional para la Prevención de Accidentes, ${ }^{4}$ observándose un descenso en el número de accidentes en el estado de 2013 a 2016.7

El objetivo de este trabajo fue definir el patrón y tipo de fracturas más frecuentes en accidentes de motocicleta atendidas en un Hospital Regional de Alta Especialidad, de Enero a Agosto de 2017, así como conocer las características de las fracturas óseas de acuerdo a la clasificación $\mathrm{AO}{ }^{8}$

\section{Material y métodos}

La población en estudio estuvo conformada por 98 pacientes que ingresaron al Área de Urgencias por sufrir un accidente en motocicleta y por presentar alguna fractura que ameritaba ingreso hospitalario y tratamiento por el Servicio de Ortopedia y Traumatología.

Criterios de inclusión: pacientes que ingresaron a urgencias por algún tipo de fractura que haya ocurrido mientras se encontraba utilizando una motocicleta, sea como pasajero o como conductor.

Criterios de exclusión: pacientes que presentaban lesiones que por sus características requerían algún otro tratamiento distinto al ortopédico o traumatológico. Pacientes que no requerían ser hospitalizados.

Consideraciones éticas: esta investigación tomó en cuenta la Norma Oficial Mexicana NOM-012-SSA3-2012, que establece los criterios para la ejecución de proyectos de investigación para la salud en seres humanos. Cada paciente firmó un consentimiento informado en el cual autorizó su participación en la investigación.

Se realizó una encuesta por paloteo (prediseñada con base en la hoja de registro de atención por violencia y/o lesión SINBA SEUL-17-P DGIS) a cada paciente que ingresó al Servicio de Urgencias Traumatológicas y que cumplía con los criterios de inclusión. Dicha encuesta fue aplicada por el médico residente en turno. El cuestionario incluye nombre del paciente, sexo, edad, diagnóstico de ingreso, la clasificación AO para los tipos de fractura, motivo del accidente o cinemática del trauma, si viajaba en motocicleta o trimoto, sitio que ocupaba el paciente en la motocicleta durante el accidente, centímetros cúbicos del vehículo, las medidas de protección utilizadas en el momento del accidente, velocidad a la que viajaba durante el accidente, si el vehículo se encontraba en movimiento, si había o no exposición de la(s) fractura(s), estado clínico que indicara el uso de alcohol o algún estupefaciente.

Clasificación AO en Traumatología y Ortopedia. En 1984, un grupo de cirujanos AO (Arbeitsgemeinschaft für Osteosynthesefragen), dirigidos por Maurice E. Müller publicaron una clasificación alfa numérica, sistematizada, que pretendía ser útil para el diagnóstico y tratamiento, así como de amplia aplicación. Se la denominó oficialmente «Clasificación de fracturas Müller AO de huesos largos». Posteriormente, continuó su desarrollo en conjunto con cirujanos de la AO y de la Asociación Estadounidense de Trauma Ortopédico (OTA) y ahora se conoce oficialmente como la «Clasificación AO/OTA de fracturas y luxaciones». ${ }^{8}$

La clasificación asigna un número a cada hueso largo. El siguiente dígito designa el segmento del hueso largo en cuestión: metáfisis proximal (1), diáfisis (2) y metáfisis distal (3), con excepción del tobillo al que se le asigna el número 4. El siguiente paso designa la morfología de la lesión, clasificándola según corresponda la lesión a un trazo simple (A), tenga un tercer fragmento (B) o sea multifragmentada (C). Esta descripción es útil únicamente en huesos largos, puesto que el resto presenta características propias y son clasificados por el mismo sistema en forma diferente (Figura 1).

Análisis de datos. Se realizó el análisis estadístico mediante el Software SSPS Windows versión 22. Para este estudio no hubo ninguna fuente de financiamiento. 


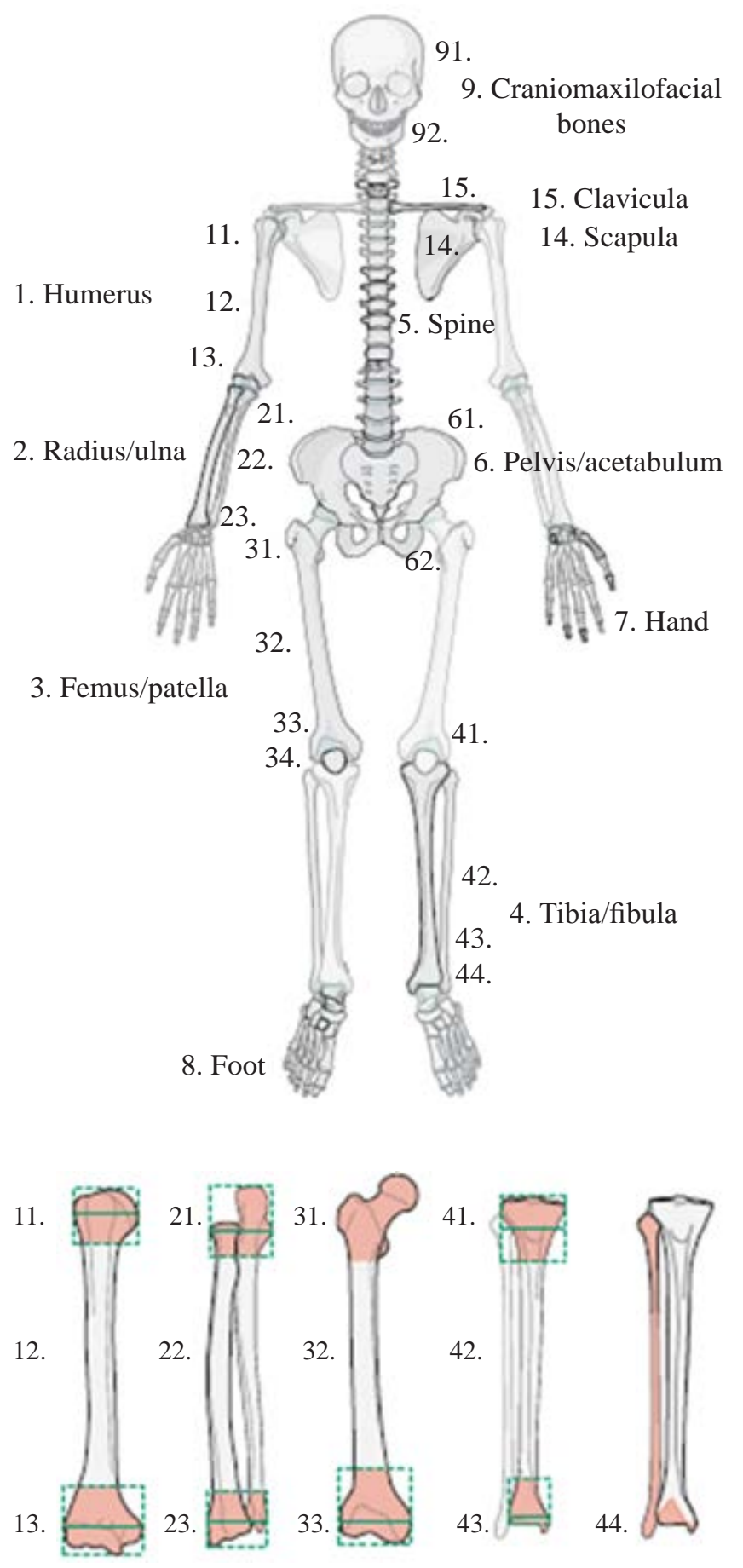

\section{Resultados}

La edad promedio de los 98 casos estudiados fue de 29.6 \pm 12.1 años. Por sexos, la distribución fue de 92 hombres y seis mujeres. La residencia de los pacientes correspondió a áreas rurales (58.1\%), suburbana (8.1\%) y urbana (33.6\%). Con relación a las lesiones observadas, hubo 141 fracturas, seis luxaciones y cinco lesiones vertebrales.

Con relación a las lesiones traumáticas observadas, la fractura más frecuente fue la de tibia y peroné (28.9\%), seguida de la fractura de fémur (25\%) y la de antebrazo (10.5\%). El resto de las fracturas representó menos de 5\%. Las fracturas expuestas correspondieron a $55.1 \%$ y las no expuestas a $44.9 \%$. La luxación más frecuente fue de la rodilla y la lesión vertebral en C4, C5 (Tabla 1 y Figura 2).

En varones, el análisis indica que las fracturas de tibia y peroné (30.5\%), fémur (24.1\%) y antebrazo (7.8\%) son las más relevantes (Figura 3). En el caso del grupo minoritario de mujeres, las lesiones más importantes fueron en antebrazo (45.4\%), fémur (36.4\%), tibia y peroné (9.1\%) y rótula (9.1\%).

De los 55 casos de fractura de tibia y peroné (tipo 4), $70.5 \%$ fueron tipo 42 (diafisaria), $22.7 \%$ tipo 41 (metáfisis proximal de tibia y peroné) y $6.4 \%$ de tipo 43 (segmento distal de la tibia). Además, se identificó la subclasificación 42B3.3 como la más usual, es decir una fractura diafisaria en tibia, con una cuña fragmentada y fractura del peroné al mismo nivel. En tibia y peroné, 52.2\% de las fracturas estaban expuestas.

En el caso del fémur, de 38 fracturas $68.4 \%$ fue de tipo 32 (diafisarias), 23.6\% fue de tipo 33 (fractura distal) y $7.89 \%$ de tipo 31 (proximal). En estas fracturas $47.36 \%$ fue expuesta. El subtipo más frecuente correspondió al 32A3.2, que es una fractura diafisaria de fémur con trazo simple, transverso, en el tercio medio diafisario.

Las fracturas del antebrazo fueron 16 y de ellas $25 \%$ fue de tipo 21 (metáfisis proximal), (25\% del tipo 22 diafisaria) y $50 \%$ del tipo 23 (metáfisis distal). Se identificó el subtipo 23A1.2 como el más frecuente, que corresponde a fractura simple de metáfisis distal del cúbito, $50 \%$ de las fracturas de antebrazo eran expuestas.

Con respecto a la cinemática del trauma de los accidentados, $60.2 \%$ sufrió daño por colisión contra un objeto en movimiento, 20.4\% por caída al derrapar, 8.2\% por choque contra un objeto fijo, $6.1 \%$ por atropellamiento por otro ve-
Anatomical location of the fracture. Anatomical location is designated by two numbers: one for the bone and one for its segment (ulna and radius as well as tibia and fibula are regarded as one bone). The malleolar segment (44-) is an exception. The proximal and distal segments of long bones are defined by a square the sides of which have the same longth as the widest part of the epiphysis (exceptions 31- and 44-).

Tomado de: AO Foundation 2018.

Figura 1: Estructura alfa-numérica de la clasificación AO/OTA mostrando el código de los huesos y su localización.
Tabla 1: Tipo de lesiones traumáticas observadas en los casos estudiados.

Tipo de lesiones observadas Casos

Fracturas

Luxaciones

Lesiones vertebrales

Total

141

5

152

Fuente: Expediente clínico. 


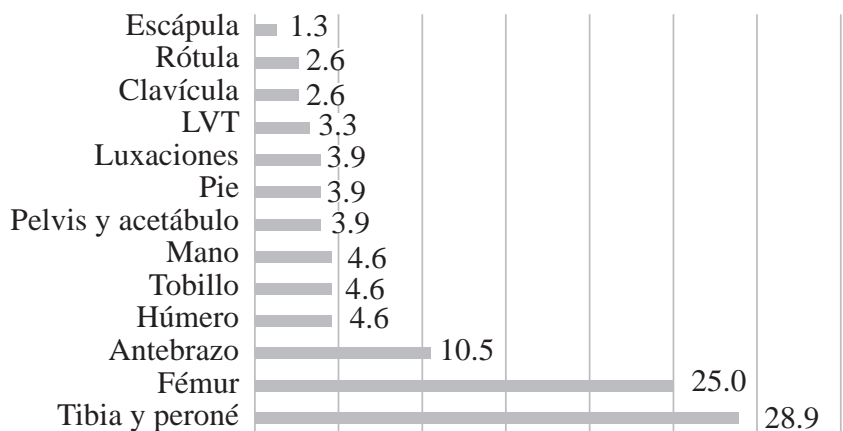

Fuente: Expediente clínico.

Figura 2: Tipo de lesiones ortopédicas. Las luxaciones incluyen lesiones cervicales y de rodilla.

hículo estando en la motocicleta y 5.1\% proporcionó datos inciertos.

El cilindraje de las motocicletas involucradas en los accidentes correspondió a menos de $150 \mathrm{~cm}^{3}$ (63), 151-250 $\mathrm{cm}^{3}$ (20) y $600 \mathrm{~cm}^{3}$ (1); en 14 casos no se obtuvo información. De los sujetos accidentados, 85 se encontraban en la posición de piloto y 10 como copilotos en la parte trasera del vehículo; en el resto de los casos no se tuvo la información precisa, $75.5 \%$ de los accidentados no utilizaba casco en el momento del accidente. Los accidentados más jóvenes constituyeron la mayoría de los que no utilizaron el casco. En 32 casos se consignaron datos clínicos que indicaban el uso de alcohol o estupefacientes por el accidentado, siendo los accidentados más jóvenes los que se encontraban bajo los efectos del alcohol o los estupefacientes.

\section{Discusión}

El evitar los accidentes y las muertes por accidente de tránsito es una de las principales metas en salud pública, ya que representan la principal causa de muerte entre personas de 15 a 29 años y una de las principales causas de incapacidad en la edad productiva, según datos de la OMS.

En este estudio, el grueso de la población captada se presentó entre los 18 y los 32 años, dato que concuerda con otros informes. ${ }^{1,6}$ Un dato relevante de esta investigación es la distribución de las edades de los pacientes, siendo el mayor porcentaje en los menores de 40 años, lo que puede significar que en los sujetos de mayor edad existen menos errores durante la conducción y por tanto los accidentes en ellos son menos frecuentes, dato que tiene concordancia con lo reportado por otros autores. ${ }^{9}$ Dos características apoyan esta relación: la cinemática del trauma, ya que en mayores de 50 años sólo dos causas estuvieron presentes, el atropellamiento y la colisión contra un objeto en movimiento; ${ }^{10} \mathrm{y}$ el hecho de que los accidentados jóvenes tienden a usar la motocicleta con mayor frecuencia bajo influjo del alcohol o de estupefacientes, además de no utilizar el casco. ${ }^{11,12}$

De acuerdo con la relación de la severidad de las lesiones y la edad, se ha reportado que a mayor edad mayor grado de lesión. ${ }^{13}$ En nuestra muestra los sujetos que presentaron traumatismo craneoencefálico (TCE) moderado y grave tenían edades menores a 37 años; las lesiones de plexo se observaron en menores de 30 años y dos tercios de los traumatismos torácicos con fracturas costales se observaron en menores de 40 años. Este hecho puede ser debido a la distribución de la curva poblacional que se captó, pero además por algunos otros factores como la conducción bajo influjo del alcohol o estupefacientes que en nuestra muestra constituyeron en su mayoría los grupos menores de 40 años (93.8\% de estos sujetos con aliento a alcohol). Asimismo, es en este grupo etario en el que mayormente no utilizaron casco como medida de protección; estos dos factores por sí mismos pueden explicar la diferencia en el patrón de lesiones con respecto a series internacionales.

En la muestra captada, sólo hubo tres mayores de 59 años. La relación descrita por Ching ${ }^{14}$ en población mayor de 65 años con respecto al mecanismo de lesión refiere que el principal mecanismo es la caída de la motocicleta; en la muestra captada en nuestro estudio, las dos cinemáticas con mayor prevalencia fueron el atropellamiento por otro vehículo y la colisión contra un objeto en movimiento. Esta diferencia puede ser debido al número de personas incluidas en este grupo etario, además de que de manera natural una persona con mayor edad presenta menor capacidad en la velocidad de reacción en un accidente.

Con respecto al empleo del casco como medida de protección, a diferencia de lo referido por otros autores, ${ }^{6}$ quienes describen que el empleo de éste osciló entre 53 y 99\%, en nuestro estudio únicamente $22.45 \%$ empleaban casco en el momento del accidente, siendo que 14 hombres no utilizaron casco por cada una de las mujeres que tampoco lo hicieron. En 2\% de los casos no se obtuvo la información respecto al uso protector del casco. El grupo etario con menor grado de protección fue de 21 a 40 años, hecho que contrasta con los resultados encontrados en población estadounidense en 2015. ${ }^{13} \mathrm{Al}$ comparar el empleo de casco y el sexo del paciente, los resultados contrastan con otro estudio realizado en 2017 en población taiwanesa, ${ }^{15}$ en el que $88.9 \%$ de las mujeres empleaban casco al momento del accidente.

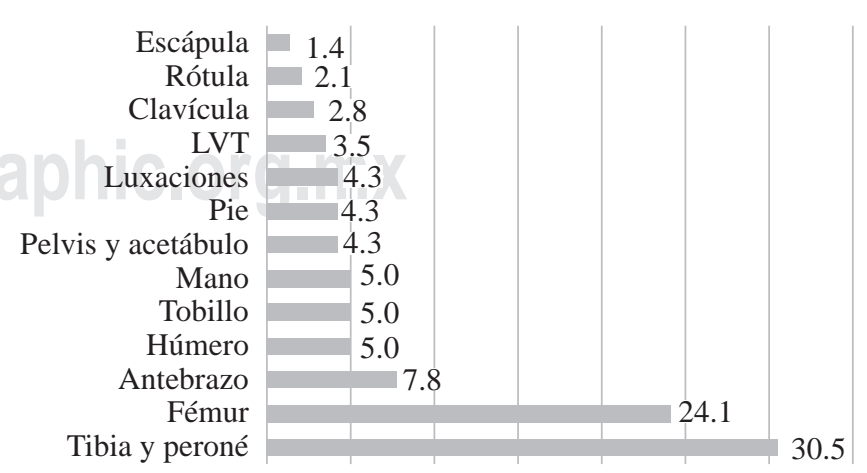

Fuente: Expediente clínico.

Figura 3: Topografía de las lesiones en hombres. 
Esta diferencia con nuestro estudio puede deberse a factores climatológicos ya que en esta región geográfica las temperaturas pueden llegar por arriba de los $40^{\circ} \mathrm{C}$; sin embargo, la ley de tránsito es clara en su normativa, requiriendo el empleo del casco como medida de protección.

Con relación al sexo de los individuos de nuestro estudio, los datos concuerdan con lo publicado en 2017 sobre población australiana ${ }^{9}$ en la que el sexo predominante fue el masculino (90\%) y en nuestro estudio fue de $93.88 \%$, dato que es concordante con los reportes estatales, pero que difiere con lo señalado por las estadísticas nacionales ${ }^{7}$ sobre accidentes de tránsito en el 2016.

El patrón de las fracturas observado de acuerdo al sexo de los pacientes muestra diferencias importantes en la distribución que pueden ser explicadas porque $50 \%$ de las mujeres viajaba como copiloto en la motocicleta y se infiere que la cinemática en la producción de la fractura aparentemente fue distinta. Además, dos pacientes presentaron múltiples lesiones, con fractura de fémur, rótula y antebrazo en una de ellas y en la otra paciente fracturas de fémur, tibia y antebrazo; siendo otro de los casos de rodilla flotante que correspondió a un paciente hombre.

Analizando la frecuencia de los conductores con aliento alcohólico o con datos clínicos de estar bajo el influjo de estupefacientes, encontramos diferencias con lo reportado por Pérez ${ }^{6}$ en 2014 quien encontró que 20\% de los conductores dio positivo a la alcoholimetría. En nuestra muestra 32.65\% de los pacientes presentó características clínicas sugestivas de estar bajo el influjo del alcohol o algún estupefaciente. Si bien, las características clínicas pueden variar en su descripción entre observadores y elevar la sensibilidad o disminuir la especificidad de dicha valoración, de cualquier forma esta puede ser útil en materia de medicina preventiva. Otro aspecto para considerar es el hecho de que casi en su totalidad los casos captados bajo el influjo del alcohol o estupefacientes pertenecen al grupo de 15 a 40 años y el 38.5\% corresponde a menores de 20 años. Este hecho nos lleva a la reflexión para la toma de medidas preventivas y normativa de tránsito en el estado, tomando en cuenta otros modelos $^{9}$ que han demostrado mayor eficacia para la disminución de la incidencia de conductores menores de 20 años bajo influjo de alcohol.

En cuanto al cilindraje de las motocicletas de los accidentes estudiados, nuestros datos concuerdan con otros reportes, ya que en el grueso de nuestra muestra correspondió a vehículos con menos de $150 \mathrm{~cm}^{3}$. Esto orienta a la búsqueda de factores de riesgo relacionados con el tipo de motocicleta, pudiéndose inferir que esta clase de vehículos es de mayor acceso a la población general y que su oferta no lleva implícito el empleo de medidas de protección o normas de tránsito específicas para los usuarios.

Con respecto al patrón de las lesiones musculoesqueléticas, existen reportes ${ }^{13}$ que hacen descripciones diferentes a las de nuestro estudio. Las más significativas son la frecuencia del sitio de lesión, refiriendo que las fracturas de tibia y peroné se encontraron en 19\% de los casos y de és- tas $34.87 \%$ fueron expuestas. En el estudio realizado, las fracturas de tibia y peroné constituyeron 28.9\% y de ellas $52.2 \%$ fueron expuestas. Esta diferencia porcentual hace diferente el pronóstico de las lesiones y el costo del tratamiento, los días de estancia hospitalaria y el material que se emplea de primera instancia en la atención de las fracturas expuestas inicialmente con fijadores externos y posteriormente en forma definitiva con clavos no fresados o bien no canulados, además del riesgo de seudoartrosis que se ve incrementado ante la exposición y la multifragmentación de los trazos, como es el caso del patrón más frecuentemente encontrado: 42B3.3 AO. Este tipo de datos no ha sido descrito en otras series.

En el caso de las fracturas de fémur, que fueron las segundas en frecuencia en nuestra serie, con el tipo 32A3.2 $\mathrm{AO}$, estas fracturas transversas se caracterizan por ser producidas por un impacto directo, en relación con el mecanismo del trauma o cinemática. En el estudio implementado fue la colisión contra un objeto en movimiento la que tuvo mayor porcentaje y que explica la frecuencia de este tipo de lesión.

En una serie de casos publicada en $2015,{ }^{13}$ las lesiones medulares representaron $16.2 \%$ y las de antebrazo $10 \%$, con $24.47 \%$ de casos con exposición. En tanto que en nuestro estudio, las fracturas de fémur fueron $25 \%$, de las cuales $47.36 \%$ eran expuestas y en segundo lugar las fracturas de antebrazo, representando $10 \%$ de los casos, la mitad de ellas expuestas. En el caso de fracturas del antebrazo, únicamente fueron considerados los pacientes cuyas fracturas requirieron tratamiento quirúrgico y hospitalización, lo que pudiera modificar la variación porcentual en la frecuencia obtenida y explicar el mayor porcentaje de fracturas expuestas.

Por otra parte, en nuestra serie las lesiones medulares ocuparon únicamente $3.3 \%$ de la muestra y de ellas las lesiones cervicales fueron las más frecuentes a diferencia de lo descrito por Burns, ${ }^{13}$ quien reportó mayor frecuencia en lesiones lumbosacras seguido de las cervicales y torácicas. Si bien nuestra muestra sólo estuvo constituida por cinco pacientes con lesiones medulares, la diferencia en el segmento de la lesión puede ser motivo de estudio.

En cuanto a las luxaciones, en la serie descrita por Burns $^{13}$ se reporta que las más frecuentes fueron las de hombro, seguidas de las del carpo. En nuestro estudio las luxaciones más frecuentes fueron las de rodilla, clasificadas como 40A, correspondiendo al 3.3\%. Esta diferencia puede ser a causa del mecanismo de lesión, considerando la cinemática del trauma, ya que el motociclista con un vehículo de cilindraje pequeño menor a $150 \mathrm{~cm}^{3}$, que es el utilizado principalmente por nuestra población, instintivamente ante una colisión tiende a bajar los pies al asfalto, produciéndose el mecanismo necesario para que se produzca la luxación de rodilla. Por otra parte, un gran porcentaje de las luxaciones de rodilla, se reducen en forma espontánea y pueden pasar subdiagnosticadas, lo que en el estudio actual puede explicar la diferencia en el porcentaje de la casuística con relación a otros estudios. ${ }^{13}$ 
El encontrar una mayor frecuencia de fracturas expuestas y rodillas flotantes en los copilotos, puede sugerir un mecanismo distinto de producción de la lesión y podría ser tomado en cuenta en las normas para la protección de este grupo. Un hecho a considerar es que aunque la muestra de pacientes femeninos captados fue bajo (seis casos), tres de ellos iban como copilotos y con patrón de fractura de fémur distal, $33 \mathrm{AO}$, lo que sugiere una contusión directa en esa región. Este dato apoya el uso de protección especial en esa región para el caso del copiloto.

\section{Conclusión}

En los accidentes en motocicleta, el grupo etario más afectado se encuentra en la población joven, productiva, del sexo masculino. La frecuencia con la que se observan fracturas expuestas está por arriba de lo documentado en el ámbito internacional. La elevada frecuencia de fracturas expuestas es en parte explicada por la carencia habitual de medidas de protección del motociclista. Los tipos de fractura más frecuentes de acuerdo con la clasificación de la AO fueron las 42B3.3, en segundo lugar el tipo 32A3.2 y en tercer lugar el tipo 23A1.2.

Referencias

1. Organización Mundial de la Salud. Lesiones causadas por el tránsito. OMS 2015. Recuperado en 2017 de http://www.who.int/mediacentre/ factsheets/fs358/es/.

2. Sandoval P. México, séptimo lugar en accidentes de tránsito. OPS, enero 2013. Recuperado en 2017 de: http://.paho.org/mex/index. php?option=com_content\&view=article\&id=552:mexico-ocupa-septimolugar-nivel-mundial-muertes-accidentes-transito-ops\&itemid $=0$.

3. Organización Mundial de la Salud. Informe sobre la situación mundial de la seguridad vial. OMS 2015, Ginebra. Recuperado en 2017 de: www.who.int.
4. Rosas OS, Cervantes TA. Los accidentes de motocicleta en México. Centro Nacional de Accidentes México: CENAPRA 3007. Recuperado en 2017 de http://conapra.salud.gob.mx/ interior/documentos/publicaciones_especializadas/los_accidentes_ motocicleta.pdf.

5. Aduayi OS, Aduayi VA, Komolate EO. Patterns of pre-hospital events and management of motorcycle-related injuries in a tropical setting. Int J Inj Contr Saf Promot. 2017; 24(3): 382-7.

6. Pérez-Núñez R, Hidalgo-Solórzano E, Vera-López JD, Lunnen JC, Chandran A, Híjar M, Hyder AA. The prevalence of mobile phone use among motorcyclists in three Mexican cities. Traffic Inj Prev. 2014; 15(2): 148-50.

7. Instituto Nacional de Estadistica y Geografia. Accidentes de tránsito terrestre en zonas urbanas y suburbanas: INEGI 2016: Psychology and Behavior 2017; 31/08/2017. http://www. inegi.org.mx/sistemas/olap/proyectos/bd/continuas/transporte/ accidentes.asp.

8. AO Foundation 2018. http://www.aofoundation.org/structure/ education/publications/classifications/pages/default.aspx.

9. Allen T, Newstead S, Lenné MG, McClure R, Hillard P, Symmons M, Dal L. Contributing factors to motorcycle injury crashes in Victoria, Australia. Transportation Research part F: Traffic Psychol Behav. 2017; 45: 157-68.

10. Chiang VX, Cheng JY, Zhang ZC, Teo LT. Comparison of severity and pattern of injuries between motorcycle riders and their pillions: a matched study. Injury. 2014; 45(1): 333-7.

11. Harvey JA, Gibreel W, Charafeddine A, Sharaf B. Helmet wear and craniofacial trauma burden: A plea for regulations mandating protective helmet wear. Chraniomaxilofac Trauma Reconstr. 2017; 10(3): 197-203.

12. Peng Y, Valdya N, Finnie R, Reynolds J, Dumitru C, Nie G, et al. Universal motorcycle helmet laws to reduce injuries: A community guide systematic review. Am J Prev Med. 2007; 52(6): 820-32.

13. Burns ST, Gugala Z, Jiménez CJ, Mileski WJ, Lindsey RW. Epidemiology and patterns of musculoskeletal motorcycle injuries in the USA. F1000 Res. 2015; 12(4): 114.

14. Hsieh LH, Liu HT, Hsu SY, Hsie HY, Chen YC. Motorcycle-related hospitalizations of the elderly. Biomed J. 2017; 40(2): 121-8.

15. Hsieh CH, Hsu SY, Hsieh HY, Chen YC. Differences between the sexes in motorcycle-related injuries and fatalities at a Taiwanese level I trauma center. Biomed J. 2017; 40(2): 113-20. 